\title{
Isolation of Polyhydroxybutyrate (PHB) Producing Bacteria, Optimization of Culture Conditions for PHB production, Extraction and Characterization of PHB
}

Christina Thapa ${ }^{1}$, Pallavi Shakya ${ }^{1}$, Rabina Shrestha ${ }^{1}$, Sushovita Pal ${ }^{1}$, Prakash Manandhar ${ }^{2}$ Department of Biotechnology, SANN International College, Purbanchal University, Nepal ${ }^{1}$ Department of Microbiology, St. Xavier's College, Nepal ${ }^{2}$

\begin{abstract}
Polyhydroxybutyrates (PHBs) are energy reserves synthesized by different micro-organisms such as Alcaligenes, Pseudomonas, Staphylococcus, Algae, in excess of carbon and limitation of nutrients like nitrogen. These biopolymers are suitable alternate to synthetic carbon-based polymers. However, the high production cost limits their commercialization. The aim of this study was thus, focused on optimization of culture condition for maximum PHB production in an attempt to reduce the production cost. The micro-organisms for this purpose were isolated from 4 different soil samples and screened for PHB production. Culture conditions for these organisms were optimized by changing the parameters, viz., incubation time, $\mathrm{pH}$, carbon source and $\mathrm{NaCl}$ concentration. Thus, optimized culture condition was used to culture the isolates for extraction of PHB and its analysis. The extracted compounds on FTIR-analysis gave characteristic $\mathrm{C}=\mathrm{O}$ peak of $\mathrm{PHB}$, thus, confirming the seven isolates to be PHB producers. Results for optimized parameters for the isolated PHB positive species showed that synthesis of PHB was maximum at 48 hours i.e. during the early stages of stationary phase. However, different isolates favored different culture conditions. Highest PHB accumulation and growth of isolates were seen at $\mathrm{pH} 7$ and 9. Similarly, it was observed that glucose was favored by 4 isolates and sucrose was favored by 3 isolates. Interestingly, $\mathrm{NaCl}$ concentration did not cause significant effect on neither the bacterial growth nor the PHB production. During the extraction of PHB from the optimized culture conditions, extraction of PHB from broth gave significant yield than that from agar. A good PHB yield from broth amounting to $36.41 \%$ and $34.59 \%$ was observed for Bacillus pasteurii and Micrococcus luteus respectively, showing a potential for their exploitation in industrial PHB production. At optimized conditions, 7 isolates exhibited significant PHB yields, thus showing a potential for further exploitation.
\end{abstract}

KEYWORDS: Bioplastics, Biopolymer, Polyhydroxybutyrates, PHB, Fourier Transform Infra-Red Spectroscopy (FTIR)

*Corresponding Author

Email: rabeenastha@gmail.com

\section{Introduction}

Traditional plastics are synthetic carbon-based polymers that are made from non-renewable source, mostly from petroleum. Due to their relatively low cost, ease of manufacture and flexibility, the demand of plastics is evergrowing. However, plastics, being man-made, are not recognized by micro-organisms. [13] Thus, they take very long to degrade i.e. 450 years on average for degradation of a plastic bottle. [7] Plastic debris also poses considerable threat by choking and starving wildlife, distributing nonnative and potentially harmful organisms, absorbing toxic chemicals and degrading to micro-plastics that may subsequently be ingested. ${ }^{22}$ Also, due to high cost of recycling, plastics are rarely recycled leading to crammed up landfills. For eradication of these and various other problems such as carbon emission during incineration, biodegradation of plastic is a must. Considerable amount of interest in the development and production of an alternative, biodegradable plastics or bioplastics is being done. Among them polyhydroxy alkanoic acids (PHAs) are drawing much attention as they have nontoxic residual products and low environmental permanence. ${ }^{\left[{ }^{9]}\right.}$ Depending on the types of carbon sources available and the biochemical pathways operating in the cell, microorganisms are capable of synthesizing various types of PHAs. Poly [R- 3hydroxybutyrate] $(\mathrm{P}[3 \mathrm{HB}])$ is the first type of PHA identified and is the most common PHA found in nature. ${ }^{[1]}$ 
PHBs are carbon and energy reserve polymers produced in bacteria, archaea, and in few eukaryotes, such as yeasts and fungi when carbon source is in plentiful and other nutrients such as nitrogen, phosphorous, oxygen or sulphur are limited. The storage molecule is then metabolized under unfavorable conditions when other common energy sources are not available. Some bacterial species which naturally produce PHB are Ralstonia eutrophes, Alcaligenes, Pseudomonas, Bacillus, Rhodococcus, Staphylococcus and Micrococcus. [1]

PHB is ecofriendly, biodegradable, biocompatible and is accumulated up to $90 \%$ of cell dry weight. ${ }^{[10]}$ PHB based plastics made by combining PHB with other biocompatible polymers (like 3-hydroxyvalerate) [8] find many applications in agriculture, packaging, and medical field including drug delivery and tissue engineering. [10] In spite of these interesting properties, industrial production of PHB is still not well established due to its high production cost. This has made it unable to compete with conventional plastics in the commercial market.

The PHB content and its composition are influenced mainly by the strain of the microorganism, the type of substrate employed and its concentration, and other growth conditions such as $\mathrm{pH}$, time and temperature. [11] Therefore, much research is needed to discover and identify novel species with vastly superior production capacity and optimization of conditions for maximal synthesis of PHB.

This research focuses on isolation and characterization of $\mathrm{PHB}$ producing bacteria from easy and convenient sources i.e. sewage soil sample. The objective was to analyze the extracted PHB by different isolated organisms in optimized physical and chemical conditions. Biochemical and morphological tests are performed for the identification purpose. In an attempt to overcome the limitations associated with costly substrates, this research is designed to use and test the efficiency of readily available and relatively cheap carbon sources such as sucrose, glucose and fructose in PHB production. Moreover, it also focuses on optimization of various growth conditions such as incubation time, $\mathrm{pH}$ and $\mathrm{NaCl}$ concentration for increase in the polymer production from respective $\mathrm{PHB}$ positive organism. Considering the optimum growth conditions and carbon source of each organism, PHB extraction and its characterization using FTIR analysis was done. The extracted PHB was calculated as percentage yield of the cell dry weight obtained.

\section{Materials and Methods}

Sample collection and isolation of pure cultures

Soil samples were collected aseptically from topsoil of four sites viz., Teku Dumping Site, Balaju Industrial Site, Banks of Dhobi Khola and Budhanilkantha Animal Waste Manure.

One gram of each sample was dispersed in $10 \mathrm{ml}$ of sterile distilled water and heated at $80^{\circ} \mathrm{C}$ for 10 minutes to isolate only endospore forming bacteria. Serial dilution of these samples was done up to $10^{-3}$, followed by spread plating of $100 \mu$ l diluted samples on nutrient agar plates. Thereafter, the plates were incubated at $30^{\circ} \mathrm{C}$ for 48 hours. Pure culture of morphologically distinct colonies was grown in modified agar plates. The constituents of Modified agar plates are: Beef extract $(0.3 \%)$, Peptone $(0.5 \%)$, Sodium Chloride (0.8\%), Glucose (1\%), and Agar (1.5\%). [12]

\section{Primary screening of PHB producing bacteria}

Detection for PHB production was employed by using lipophilic stain Sudan Black B. ${ }^{[3]}$ Stain was prepared by dissolution of $0.3 \mathrm{gm}$ powdered stain in $100 \mathrm{ml}$ of $70 \%$ ethanol. For microscopic studies, smears of colonies were heat-fixed on clean, grease-free glass slides, followed by staining with $0.3 \%$ solution of the Sudan Black B. After leaving the slides undisturbed for 15 minutes, immersion in xylene and counterstaining with safranin $(5 \% \mathrm{w} / \mathrm{v}$ in sterile distilled water) was performed. Cells appearing blue-black under microscope were accredited as PHB positive strains.

PHB positive strains were preserved on two vials, viz., working and stock vials, containing agar slants with $2 \%$ glycerol for preservation. 


\section{Morphological and Biochemical Characterization of $\mathrm{PHB}$ positive Isolates}

Distinct morphological features of the isolates were recorded on the basis of shape, color and size. Similarly, cellular morphology was studied under the microscope using Gram Staining and Endospore Staining. Standard microbiological methods were employed for identification of isolated bacteria by biochemical tests. The tests performed were IMViC test, nitrate test, sugar utilization test, catalase test, oxidase test, starch utilization test and oxidative-fermentative test.

\section{Growth Curve Study of Isolates}

PHB producing medium was used to study the growth and production of PHB. The components of the media are: Glucose - 1g, Peptone -

$0.25 \mathrm{~g}$, Yeast extract $-0.25 \mathrm{~g}, \mathrm{NaCl}-0.01 \mathrm{~g}$, $\mathrm{KH} 2 \mathrm{PO} 4-0.05 \mathrm{~g}, \mathrm{MgSO} 4-0.02 \mathrm{~g}$ and $\mathrm{pH}$ at 7. [6]

One percent inoculums from activated $\mathrm{PHB}$ positive isolates were inoculated in conical flasks containing PHB producing media, followed by incubation of the culture for 48 hours at $37^{\circ} \mathrm{C}$ with occasional shaking. At an interval of every 4 hours, the samples were collected to perform Sudan staining and the biomass reading was done using spectrophotometer at $640 \mathrm{~nm}$.

\section{Optimization of PHB production Effect of $\mathrm{pH}$}

Every microorganism has a minimum, an optimum and a maximum $\mathrm{pH}$ for growth. To standardize the optimum $\mathrm{pH}$ for the production of $\mathrm{PHB}$, the $\mathrm{PHB}$ positive bacterial cultures were inoculated in PHB producing media at different $\mathrm{pH}\left(2,4,7,9\right.$ and 11) and incubated at $37^{\circ} \mathrm{C}$ for 48 hours with occasional shaking. The $\mathrm{pH}$ values were taken in order to cover different acidic, neutral and basic $\mathrm{pH}$ ranges. After incubation, the samples were screened using Sudan stain to confirm PHB production and turbidity of the media due to bacterial growth was measured by spectrophotometer at $640 \mathrm{~nm}$.

\section{Effect of $\mathrm{NaCl}$ Concentration}

Microorganisms vary widely in their $\mathrm{NaCl}$ tolerance. Thus, PHB producing media with different $\mathrm{NaCl}$ concentrations $(0.1 \%, 0.5 \%, 2 \%$, $5 \%$, and $10 \%$ ) was prepared. After autoclaving, $1 \%$ of activated culture was added to each tube and incubated at $37^{\circ} \mathrm{C}$ for 48 hours. The samples were collected after 48 hours for Sudan Staining and for measurement of O.D. at 640nm.

\section{Effect of Carbon Sources}

$2 \%$ glucose, sucrose, and fructose were added into PHB producing media as carbon sources and the selected isolates were grown in it. After incubation and screening by Sudan stain, the PHB produced by the isolates was quantified spectrophotometrically for the selection of carbon source that showed highest PHB production.

\section{Extraction of PHB}

The optimized $\mathrm{pH}$ and carbon source for each bacterium were used for the extraction of PHB by solvent extraction method [4] with slight modifications. Firstly, 1\% of PHB positive strain was inoculated in PHB producing media of optimized $\mathrm{pH}$ and carbon source and it was incubated at $37^{\circ} \mathrm{C}$. After each 4-hour, $1 \mathrm{ml}$ of media was centrifuged at $11,800 \mathrm{rpm}$ for 20 minutes and Sudan staining was done to confirm PHB production. When the PHB production was confirmed, which mostly ensued after 48 hours, $50 \mathrm{ml}$ of bacterial cell culture growth was taken and pelleted at 5000 rpm for 25 minutes. The dry weight of the pellet was taken and then it was washed with acetone and ethanol successively. For the recovery of $\mathrm{PHB}$, equal volume of $6 \%$ sodium hypochlorite was used to re-suspend the pellet and it was incubated at $37^{\circ} \mathrm{C}$ for 10 minutes. This was followed by centrifugation at $5000 \mathrm{rpm}$ for 30 minutes to sediment the lipid granules. The pellet obtained was washed with acetone and ethanol followed by hot chloroform treatment. After the pellet dissolved in chloroform, Whatman filter paper was used to filter out the cell residues so that only PHB is present in the chloroform solution. Finally, the filtrate was evaporated in hot air oven at $40^{\circ} \mathrm{C}$ and dry weight of extracted PHB was measured. The percentage of $\mathrm{PHB}$ accumulation was calculated using the formulae: 


$$
\frac{\text { Dry weight of extracted } P H B\left(\frac{g}{m l}\right) \times 100}{\text { Dry weight of biomass }}
$$

strains inoculated in broth media as well as in
This procedure was repeated for all the PHB positive strains and $\mathrm{PHB}$ was extracted from the was found that five of the strains belonged to

Table 1. Morphological Characteristics of Strains

\begin{tabular}{llllllll}
\hline Morphology & T101 & B139 & Y202 & K302 & X102 & D301 & L402 \\
\hline Gram's Test & + & + & + & + & + & + & + \\
Cell Size & L: $1 \mu \mathrm{m}$ & L: $3 \mu \mathrm{m}$ & Diameter: & Diameter: & L: $5 \mu \mathrm{m}$ & L:5 $\mu \mathrm{m}$ & L: $5 \mu \mathrm{m}$ \\
& B:2 $\mu \mathrm{m}$ & B: $1 \mu \mathrm{m}$ & $2 \mu \mathrm{m}$ & $2 \mu \mathrm{m}$ & B:1 $\mathrm{m}$ & B:1 $\mu \mathrm{m}$ & B: $1 \mu \mathrm{m}$ \\
Shape & Rod & Rod & Cocci & Cocci & Rod & Rod & Rod \\
Spore & + & + & - & - & + & + & + \\
Margin & Irregular & Smooth & Smooth & Smooth & Wooly & Smooth & Smooth \\
Color & White & Light & Yellow & Yellow & Pinkish White & White & White \\
Elevation & Flat & Yellow & & & & Flat & Convex \\
Opacity & Translucent & Opaque & Opaque & Opaque & Translucent & Opaque & Opaque \\
\hline
\end{tabular}

agar media.

\section{Characterization of PHB}

The presence of prominent functional groups such as $\mathrm{CH}_{3}, \mathrm{CH}_{2}, \mathrm{C}=\mathrm{O}, \mathrm{C}-\mathrm{O}, \mathrm{CH}$, and $\mathrm{OH}$ is a critical decider of presence of PHB. ATR-FTIR spectroscopy (Spectrum65 FTIR spectrometer) was used for qualitative identification and for checking the presence of such groups in the extracted compounds.

Table 2. Identification of Strains

\begin{tabular}{|c|c|c|}
\hline \multirow[b]{2}{*}{ S.N. } & \multirow[b]{2}{*}{ Strain } & Identification from Bergey's \\
\hline & & $\begin{array}{l}\text { Manual of } \\
\text { Bacteriology }\end{array}$ \\
\hline 1. & T101 & Bacillus pumilus \\
\hline 2. & B139 & Bacillus megaterium \\
\hline 3. & Y202 & Arthrobacter sp. \\
\hline 4. & K302 & Micrococcus luteus \\
\hline 5. & X102 & Bacillus pasteurii \\
\hline 6. & D301 & Bacillus cereus \\
\hline 7. & L402 & Bacillus sphaericus \\
\hline
\end{tabular}

\section{Result and Disscussion Isolation and Screening}

Altogether, 23 colonies, which were distinct, were chosen based on their shapes and colors. After 24-48 hours culture period, Sudan Black B staining was done to confirm the presence of PHB granules. Among 23 bacteria, 7 were found to be Sudan positive, i.e. they were capable of producing lipid granules which could have the presence of PHB.

\section{Morphological Characterization}

The result of morphological and biochemical characterization of the 7 Sudan positive bacteria is shown in Table 1 and Table 2 respectively. It
Bacillus species and 2 strains, Y202 and K302, belonged to Arthrobacter species and Micrococcus luteus respectively.

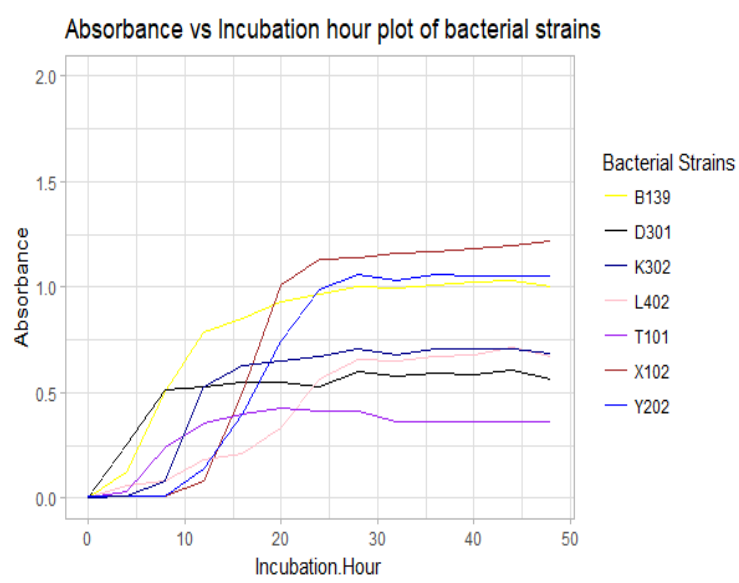

Figure 1: Graph showing growth curve of isolates It was found that high amounts of black stained granules were obtained in the 48-hour period for all the bacteria. Therefore, 48 hours was chosen as the optimized incubation time for PHB production.

\section{Growth Curve Analysis}

The inoculated cultures were incubated at $37^{\circ} \mathrm{C}$ for 48 hours and readings were taken at $640 \mathrm{~nm}$ using spectrophotometer. $640 \mathrm{~nm}$ was taken as the required wavelength because the Wavelength of Measurement (WM) of OD depends on growth of the culture and here, we expect higher growth.

The Absorbance vs. Incubation hour plot of bacterial strains plotted using R-programming has been shown in the Figure 1.

\section{Optimization of Culture Conditions}

The 7 strains of Sudan positive bacteria were subjected to growth in the $\mathrm{PHB}$ producing media prepared with different $\mathrm{pH}, \mathrm{NaCl}$ and 
carbon sources. The graphs of Absorbance vs the optimized conditions of bacterial strains were plotted using R-programming (Figure 4, 5 and 6 respectively).

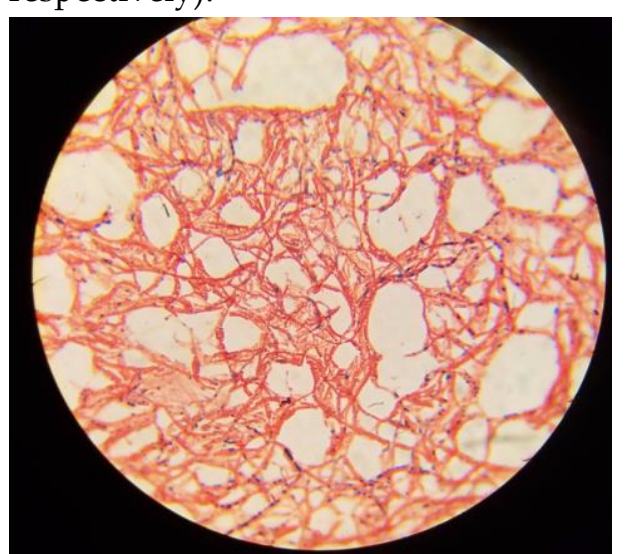

Figure 2: Sudan Stained black PHB granules of strain L402

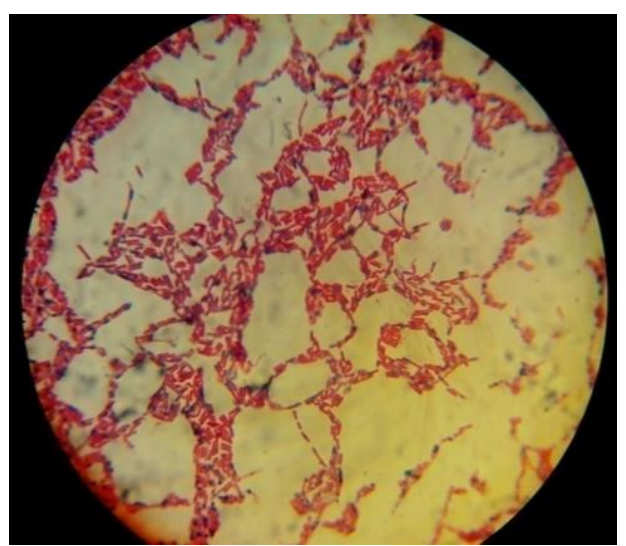

Figure 3: Sudan Stained black PHB granules of strain D301

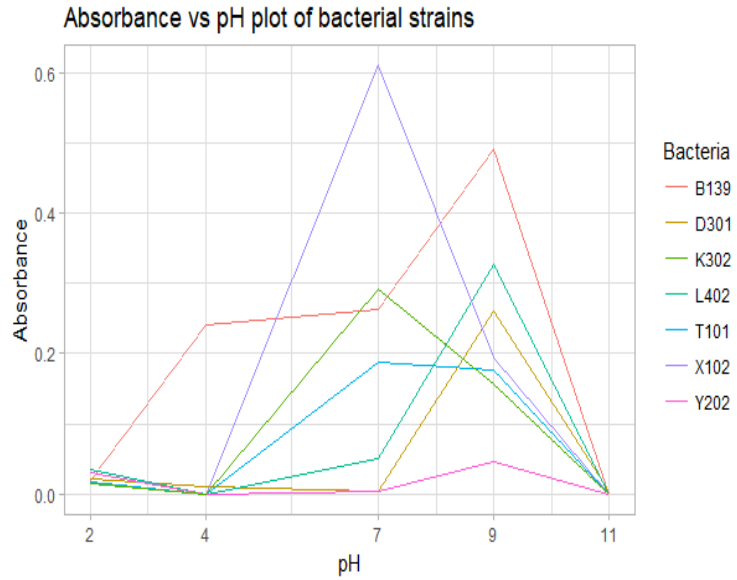

Figure 4: Graph showing absorbance vs. pH plot of bacterial strains. It was established that, for Bacillus megaterium (B), Arthrobacter sp.(Y), Bacillus cereus (D) and Bacillus sphaericus (L) the optimized $\mathrm{pH}$ was 9, for Micrococcus luteus (K) and Bacillus pasteurii (X), the optimized $\mathrm{pH}$ was 7 and for Bacillus pumilus (T), pH 7 and $\mathrm{pH} 9$ were compatible. Thus, we can deduce that acidic $\mathrm{pH}$ is not suitable for $\mathrm{PHB}$ production.

\section{Extraction of PHB}

Extraction was performed from both PHB producing broth and agar. It was observed that extraction from broth gave much better results than that from agar from all of the species except Arthrobacter spp. The highest percentage of PHB accumulation from broth culture was shown by Bacillus pasteurii and the lowest was shown by Arthrobacter spp. Similarly, from agar,

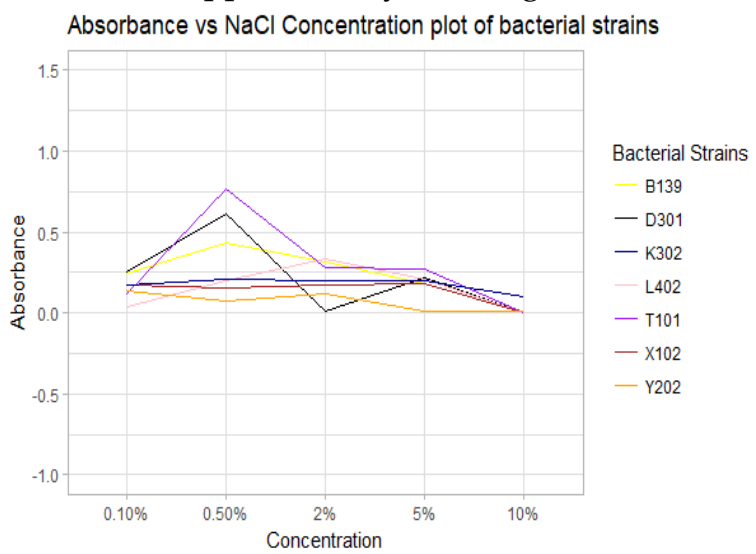

Figure 5: Graph showing absorbance vs. $\mathrm{NaCl}$ Concentration plot of bacterial strains. It was established that Bacillus pumilus(T), Bacillus megaterium (B) and Bacillus cereus (D) grew the most in the concentration of $0.5 \%$, Arthrobacter spp. $(\mathrm{Y})$ and Bacillus sphaericus (L) grew the most in the concentration of $2 \%$ while Micrococcus luteus (K) and Bacillus pasteurii (X) grew uniformly in all the concentrations except in $10 \%$.

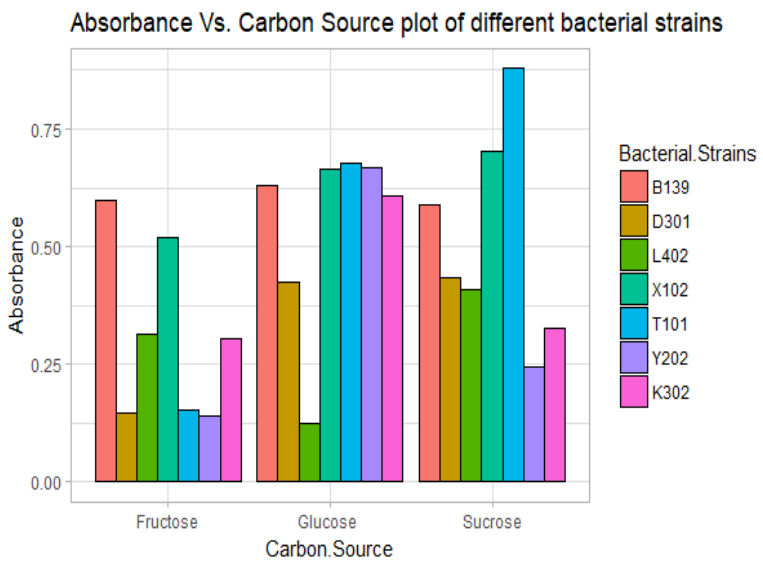

Figure 6: Graph showing effect of different carbon sources on bacterial growth. It was observed that Bacillus species could grow and produce PHB in both Glucose and Sucrose. Bacillus pumilus (T), Bacillus pasteurii $(\mathrm{X})$ and Bacillus sphaericus preferred Sucrose while Bacillus megaterium (B) preferred glucose and Bacillus cereus preferred both Glucose and Sucrose. Arthrobacter spp (Y) and Micrococcus luteus (X) preferred glucose.

Arthrobacter spp. showed highest accumulation of PHB. Bacillus pasteurii, Bacillus pumilus, Bacillus sphaericus did not show any growth in 
agar possibly due to limited availability of nutrients in their respective growth conditions.

Table 3. Comparison between percentage PHB accumulation from isolates grown in broth and

Table 3. Comparison between percentage PHB accumulation from isolates grown in broth and agar

\begin{tabular}{lllll}
\hline S.N. & Bacterial Code & Bacteria & $\begin{array}{l}\text { \%PHB } \\
\text { from broth }\end{array}$ & $\begin{array}{l}\text { accumulation } \\
\text { from agar }\end{array}$ \\
\hline 1. & X102 & Bacillus pasteurii & 36.41 & - \\
2. & K302 & Micrococcus luteus & 34.59 & 17.65 \\
3. & B139 & Bacillus megaterium & 28.63 & 4.12 \\
4. & T101 & Bacillus pumilus & 21.46 & - \\
5. & L402 & Bacillus sphaericus & 18.45 & - \\
6. & D301 & Bacillus cereus & 14.91 & 9.35 \\
7. & Y202 & Arthrobacter spp. & 8.56 & 20.65 \\
\hline
\end{tabular}

\section{FTIR Analysis}

The functional groups of extracted PHB were identified using FTIR Analysis. The functional groups of PHB extracted from Bacillus pasteurii, Arthrobacter spp., Micrococcus luteus and Bacillus cereus was confirmed as $\mathrm{C}=\mathrm{O}$ groups.

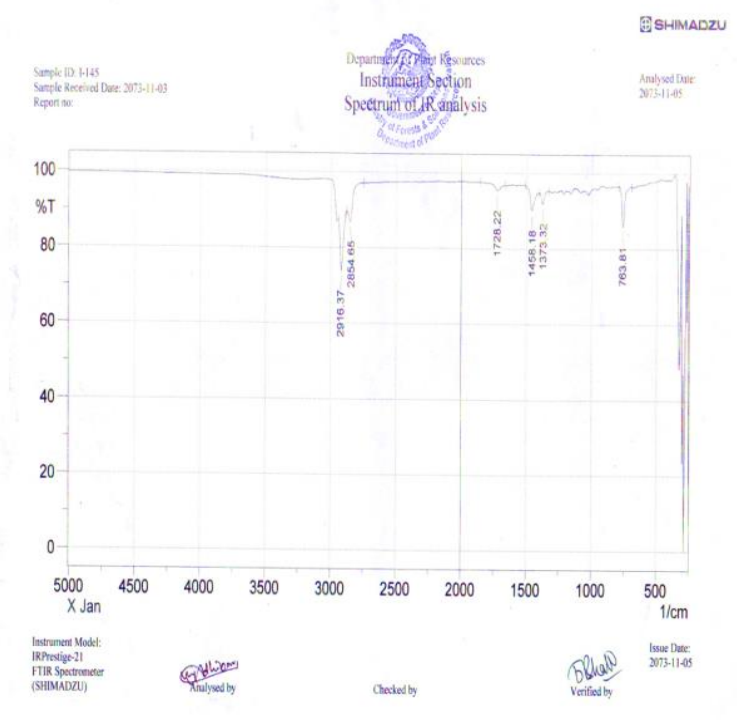

Figure 7: FTIR Analysis of extracted product from X102 (Bacillus pasteurii)

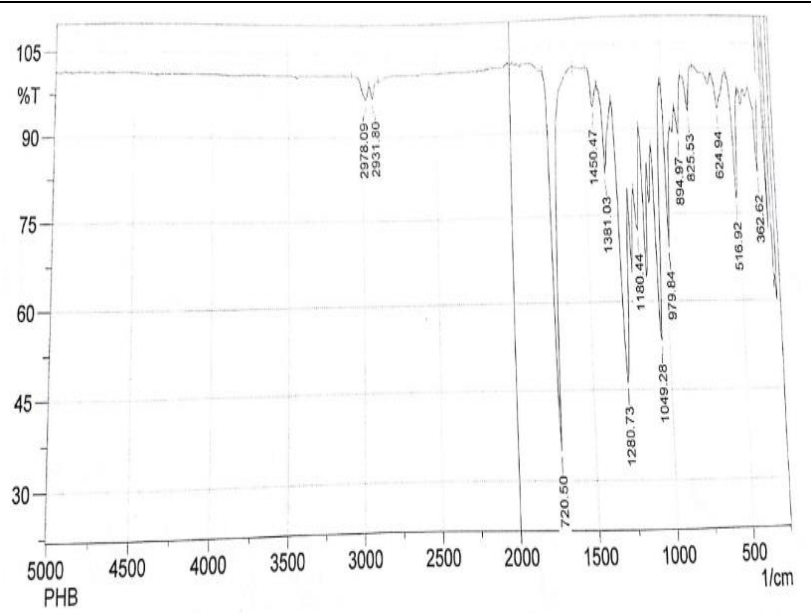

Figure 8: FTIR Analysis of Standard PHB

\section{Conclusion}

The present study was designed for the isolation of effective poly-hydroxybutyrate producing strains from soil to yield maximum PHB under optimized conditions. From our research, we found out that cosmopolitan "Everything is Everywhere" population such as Bacillus, Arthrobacter and Micrococcus species were able to produce PHB in considerably good quantity compared to other isolated species. Consequently, the effect of various parameters like carbon source, incubation time, $\mathrm{pH}$ and $\mathrm{NaCl}$ concentration on $\mathrm{PHB}$ production were seen to be species specific. Similarly, the production from broth and fermentation methods gave much better results than that from agar in all of the isolated species except Arthrobacter. PHB yield from broth amounting to $36.41 \%$ and $34.59 \%$ was observed in Bacillus pasteurii and Micrococcus luteus respectively, showing a potential for their exploitation in 
industrial PHB production. Further characterization of extracted products with the help of ATR-FTIR analysis showed prominent functional groups $\mathrm{CH}_{3}, \mathrm{CH}_{2}, \mathrm{C}=\mathrm{O}, \mathrm{C}-\mathrm{O}, \mathrm{CH}$ and $\mathrm{OH}$, which when compared with the standard PHB curve, confirms the extracts as PHB. Hence, this project focused on the isolation of microorganisms from soil samples of polluted sites and the optimization of conditions for the production of PHB effectively and frugally.

\section{Acknowledgement}

We sincerely thank the biotechnology department and management of SANN International College for funding our research work. We are also grateful to Mrs. Manjushree Hada for her constant support and supervision.

\section{References}

1. Ansari S, Fatma, T: Polyhydroxybutyrate - a Biodegradable Plastic and its Various Formulations. International Journal of Innovative Research in Science, Engineering and Technology 2014, 3(2):9494-9499.

2. Barnes DKA, Galgani F, Thompson RC, Barlaz M: Accumulation and Fragmentation of Plastic Debris in Global Environments. Philosophical Transactions of the Royal Society B: Biological Sciences 2009; 364(1526): 1985-1998.

3. Burdon KL, Stokes JC: Studies of the common aerobic spore-forming Bacilli staining for fat with Sudan Black B stain. Journal of Bacteriology 1942, 43: 717-724

4. Chang Y, Hahn S, Kim B, Chang H: Optimization of Microbial Poly (3 hydroxybutyrate) Recovery Using Dispersions of Sodium Hypochlorite Solution and Chloroform. Biotechnol. Bioeng. 1994, 44(2): 256-261.

5. Godale C, Ambarshetti S: Media optimization for $\mathrm{Phb}$ production and its application as precursor for bioplastics. European Journal of Biotechnology and Bioscience 2015, 3(12):49-51.

6. Mikkili I, Karlapudi AP, Venkateswarulu, Nath SB, Kodali VP: Isolation, Screening and Extraction of Polyhydroxybutyrate (PHB) producing bacteria from Sewage sample. International Journal of PharmTech Research 2014,6(2): 850-857
7. LeBlanc R: How Long Does It Take Garbage to Decompose? the balance 2017.

8. Nehra K, Chhabra N, Sidhu P, Lathwal P, Rana J S: Molecular identification and characterization of Poly- $\beta$-hydroxybutyrate (PHB) producing bacteria isolated from contaminated soils. Asian Journal of Microbiology, Biotechnology and Environmental Sciences 2017, 17:281-290.

9. Pachekoski WM, Agnelli JAM, Belem LP: Thermal, mechanical and morphological properties of poly (hydroxybutyrate) and polypropylene blends after processing. Materials Research 2009, 12(2):159-164

10. Rydz J, Wanda S, Mariya K, and Darinka C: Polyester-Based (Bio) Degradable Polymers as Environmentally Friendly Materials for Sustainable Development. International Journal of Molecular Sciences 2015, 16(1): 564-596.

11. Saharan BS, Grewal A, Kumar P: Biotechnological Production of Polyhydroxyalkanoates: A Review on Trends and Latest Developments. Chinese Journal of Biology 2014.

12. Singh G, Mittal A, Kumari A, Goel A, Aggarwal NK, Yadav A: Optimization of Poly-B-Hydroxybutyrate Production from Bacillus species. European Journal of Biological Sciences 2011, 3 (4): 112-116.

13. Wolchover N: Why Doesn't Plastic Biodegrade? LiveScience 2011. 